\title{
Development of optical-electronic systems for the study of temporal trends in the atmosphere composition and temporal variations of the Earth's climate
}

\author{
N I Moskalenko, S N Parzhin, I R Dodov*, M S Hamidullina and A R Akhmetshin \\ Kazan State Power Engineering University, Russia, 420066, Kazan, Krasnoselskaya, 51
}

\begin{abstract}
Optical-electronic system for atmospheric composition registration is considered. It is intended to identify the influence of anthropogenic emissions into the atmosphere on its optical properties and the Earth's climate on a planetary scale. Anthropogenic emissions into the atmosphere increase due to the growth of planet population and expansion of its economic activity. The increasing scale of influence of various ingredients of anthropogenic emissions on the atmosphere requires studying their optical properties and carrying out research on the atmospheric composition in order to identify temporal trends in the concentrations of small and trace components of the atmosphere and their influence on changes in the Earth's climate.
\end{abstract}

\section{Introduction}

This work aims to obtain baseline data for the developers of optical-electronic instruments for recording the composition of anthropogenic emissions and studying optical characteristics of various ingredients in a wide spectral range of $0.1-100 \mu \mathrm{m}$. The developed modeling complexes described in [1-4] can serve as prototypes of more modern optical-electronic systems designed to control the gas and dispersed ingredient composition of the atmospheric emissions phases by industrial enterprises, energy enterprises, vehicles, aeronautics, as a result of agricultural activity and cattle breeding. Due to warming in the Arctic zone, permafrost melting occurs, which causes gas release to the atmosphere. Testing of the developed complexes [1-5] with obtaining positive results in vacuum ultraviolet (VUV) and far infrared regions provides the prospect of creating operating measuring complexes of an extended spectral range. Currently obtained results on the impacts of anthropogenic emissions into the atmosphere of optically active small and trace ingredients indicate a temporary trend of increasing their concentration which leads to global climate warming on Earth and especially in the Arctic zone.

\section{Laboratory measuring equipment complexes for the study of radiation characteristics of gas components of combustion products and radiation absorption by atmospheric gases}

This work is based on the results of experimental studies carried out in NPO GIPO, LLC SB TEXA on spectrometry and spectroradiometry of products of gaseous fuel combustion in a wide spectral range of 0.250 microns. It goes into a shorter region of the VUV spectrum, so it requires revision of the developed equipment complexes and modernization of individual components of the measuring complexes. The UV spectrum should be unreservedly explored in order to develop optical-electronic devices for monitoring of environment, the state of rooms' environment pollution by toxic products, the economic activity, the composition of natural gases toxic emissions of combustion products into the atmosphere, and the gas composition in natural gas processing lines.

Expansion of the field of research into VUV region leads to serious difficulties associated with the need to evacuate all measuring equipment; the lack of transparent materials for protective windows; the lack of commercially available vacuum spectral equipment of increased spectral resolution; the complete absence of industrial radiation sources required for energy calibration. The main absorption components of air in VUV region are molecular gases: nitrogen, ozone, and oxygen. To completely eliminate the influence of atmospheric air absorption in laboratory installations and

Corresponding author: utisey@gmail.com 
spectral instruments, it is necessary to achieve vacuum of the order of $10^{-2}-10^{-3} \mathrm{~Pa}$.

In the present work the ingredient composition of the investigated medium is determined using the method of spectral analysis of high resolution spectra [7-9] and the method of spectral analysis of atmospheric compression at elevated pressures of 20-70 atm, which increase the limiting sensitivity of the measured ingredient concentration. Measurements of spectral absorption at elevated pressures of compressed atmosphere in multipasscell (MPC) provide ingredients concentration $\rho_{i}$ determination with a relative error of $\triangle \rho_{i} / \rho_{i}=3-4 \%$.

To determine the temporal trends in changes of $\mathrm{CH}_{4}$, $\mathrm{CO}_{2}, \mathrm{CO}, \mathrm{N}_{2} \mathrm{O}$ concentrations, the results of measurements on spectral complexes with MPC-1, MPC-2, MPC-4, MPC-6 were used.

The measuring complex with MPC-4 was specially created to measure the time trends of changes in the concentrations of optically active ingredients. It is a multi-pass cell with a variable base of $4,8,16$ meters, installed on an open pavilion in a pine forest. It allows one to model optical paths with a length from 16 meters up to $1 \mathrm{~km}$ with a pressure in MPC-4 from $10^{2} \mathrm{~Pa}$ to $2 * 10^{6} \mathrm{~Pa}$ in spring, summer, and autumn. In winter measuring complexes with MPC-1 [7], MPC-2 [4], MPC-6 [7] were used. The spectral measuring complex MPC-6 with a high-aperture cryogenic cuvette [7] makes it possible to perform measurements with high spectral resolution and at low temperatures up to $200 \mathrm{~K}$ in combination with the DSL-1 or the IKM-731 spectrometers. To measure the $\mathrm{CO}_{2}$ concentration in the atmosphere a two-beam MPC was used in combination with IKS-24 spectrometer [3] with a spectral resolution limit of $\Delta v=0.5 \mathrm{~cm}^{-1}$.

To determine the ingredient composition from the absorption spectra of high spectral resolution we used mathematical processing of the medium absorption spectrogram by a quintic spline. The obtained spectrum of optical thickness is further divided by the method of differential moments into individual contours of individual spectral lines with subsequent restoration to the influence of the apparatus function of the spectrometer. The ingredients are identified according to the position of spectral line centers. The ingredient concentration is determined using optical thickness of the spectral line center, length of the optical path in the MPC and the known intensity of the spectral line [9].

Fig. 1 presents the measurements results of methane concentration in the atmosphere and the temporal trend of methane concentration in the period from 1964 to 2015 obtained by the method of atmospheric compression and from the solar spectra of high spectral resolution. The methane concentrations measured by different methods correlate well with each other and are consistent with the results of measurements presented in [10]. An increase in concentration over time was observed for $\mathrm{N}_{2} \mathrm{O}$ and $\mathrm{CO}_{2}$ [10].

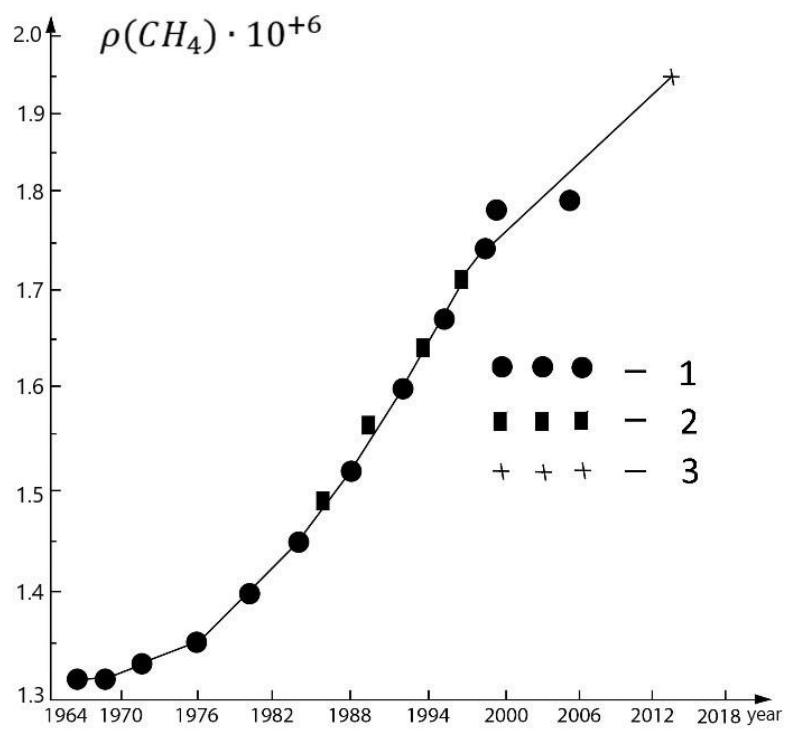

Fig. 1. Temporal trend of $\mathrm{CH} 4$ concentration change: 1 - data obtained by the method of atmosphere compression; 2 measurements by solar spectra; 3 - data from [10].

In $[11,12]$, the modeling of the greenhouse effect of the Earth's atmosphere was developed and for the global greenhouse effect of methane the following relationship was obtained [12]:

$$
\triangle \mathrm{T}\left(\mathrm{CH}_{4}\right)=0.74 *\left(\triangle \mathrm{CH}_{4} / \mathrm{CH}_{04}\right)^{0.57},
$$

where $\mathrm{CH}_{04}$ is the methane concentration in 1964, $\triangle \mathrm{CH}_{4}$ is the temporary change in methane concentration, 0.74 is the greenhouse effect $(\mathrm{K})$, caused by methane in 1964 . The increase in the greenhouse effect of small atmospheric components causes an increase in the Earth's temperature as a planet and the concentration of $\mathrm{H}_{2} \mathrm{O}$ vapor in the atmosphere which causes an additional greenhouse effect of $\mathrm{H}_{2} \mathrm{O}$ vapor taking into account the correlation. This connection of the greenhouse effect of $\mathrm{CH}_{4}$ with the greenhouse effect of $\mathrm{H}_{2} \mathrm{O}$ vapors leads to an increase in the surface temperature change by a factor of 2.1 [11].

Thus the mean global contribution to the change in surface temperature from 1964 will be $\mathrm{T}(\mathrm{CH} 4)=2.1 * 0.74 *\left(\triangle \mathrm{CH}_{4} / \mathrm{CH}_{04}\right)^{0.57}, \mathrm{~K}$.

When calculating the greenhouse effect of atmospheric $\mathrm{CH}_{4}$ and $\mathrm{N}_{2} \mathrm{O}$ it is necessary to take into account the absorption of solar radiation in the near-IR spectral range which is determined by the spectral absorption coefficients obtained from the experimental spectra shown in Figs. 2, 3 at high pressures.

Fig. 4 illustrates the results of determining the distribution function $f(r)$ of the number of particles in size for the combustion products of gasoline and diesel engines from the results of measurements of the spectral optical attenuation characteristics of the radiation by the dispersed phase of the combustion products of bus transport. Normalization of this function is $\int f(r) d r=1$, where $r$ is the radius of the black sol particles. In [7] data on measurements of the distribution of the number of particles by size $f(r)$ in the combustion 

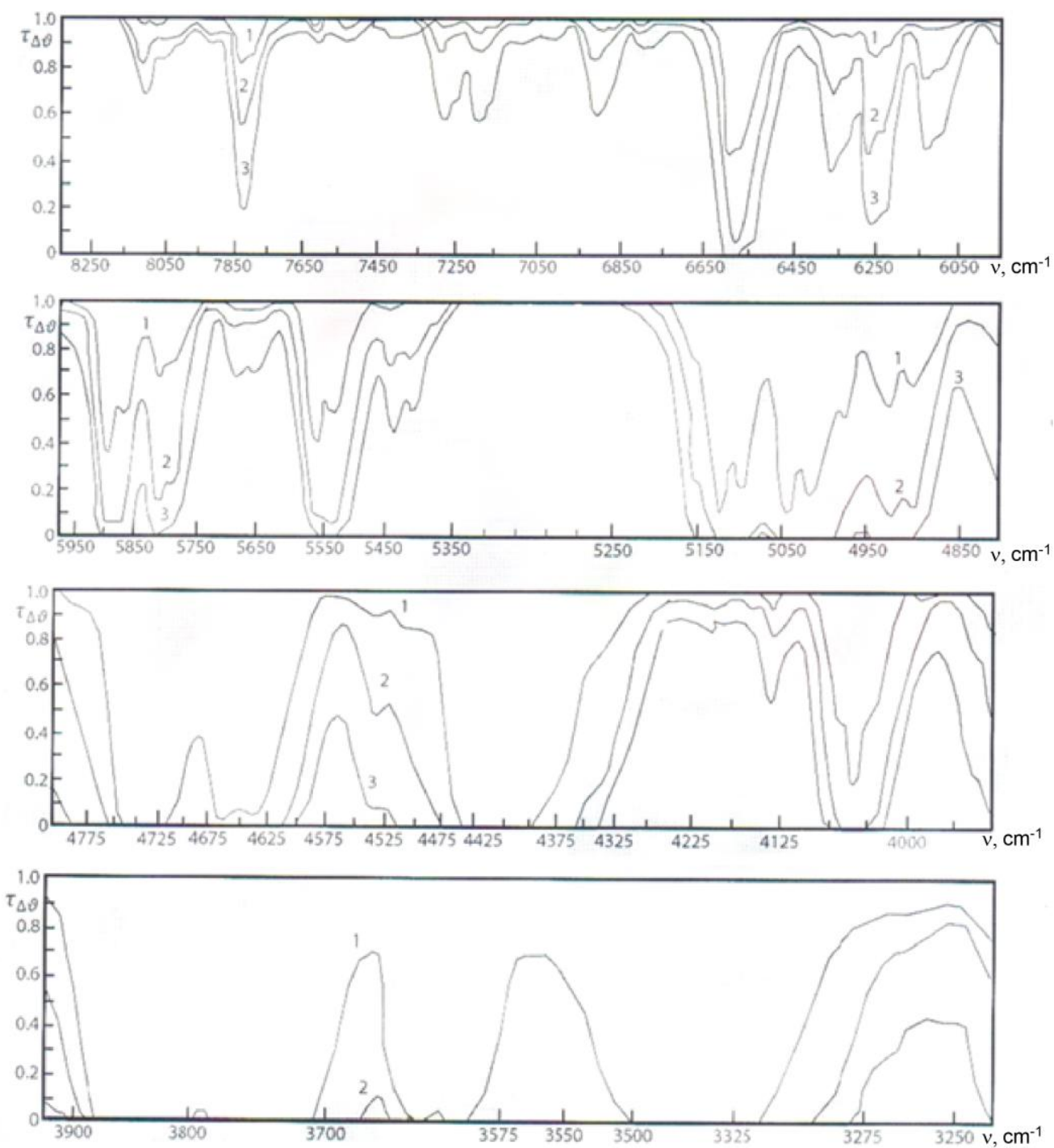

Fig. 2. The measured absorption spectra of nitrous oxide in the spectral region of $325-8250 \mathrm{~cm}^{-1} \mathrm{P}\left(\mathrm{N}_{2} \mathrm{O}\right)$ : $1-2 ; 2-10 ; 3-25.6$ atm; $\mathrm{L}=10 \mathrm{~m} ; \mathrm{T}=300 \mathrm{~K}$.

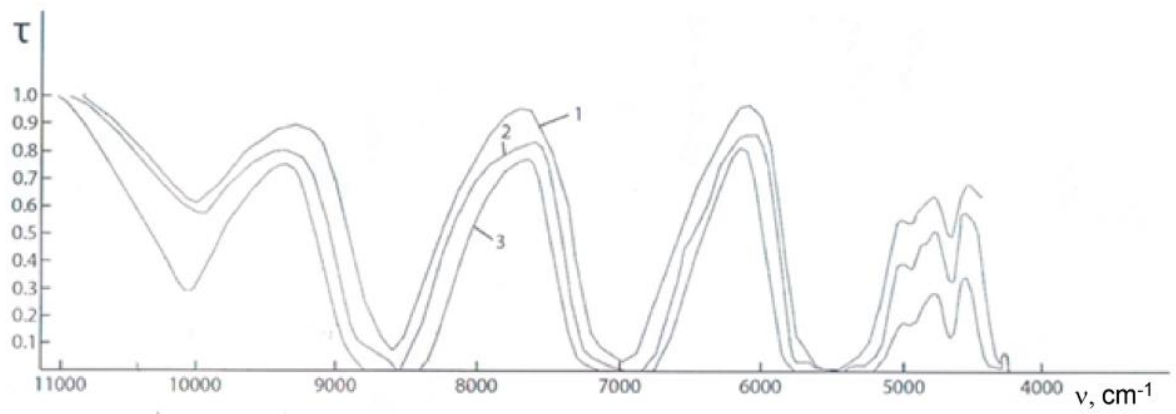

Fig. 3. Experimental absorption spectra of technical methane at high absorbing masses. $\mathrm{W}\left(\mathrm{CH}_{4}\right)$ : 1-2560, 2-3840, 3-7680 atm. cm; $\mathrm{P}\left(\mathrm{CH}_{4}\right)$ : 1-0.4, 2-1.2, 3-1.2 atm; L: 1-64; 2-32; 3-64 m.

products of gaseous energy fuels of different chemical composition are presented. In [7] calculations of radiation scattering matrices by soot particles were performed and the spectral dependences of the attenuation absorption radiation scattering coefficients in the spectral range $0.2-50 \mu \mathrm{m}$ were determined.
For different distributions $f_{i}(r)$ the real distribution $f$ (r) can be represented as $f(r)=\sum_{i} A_{i} f_{i}(r)$ where $A_{i}$ is the weight of each fraction of the sol in the total distribution $f(r)$. 


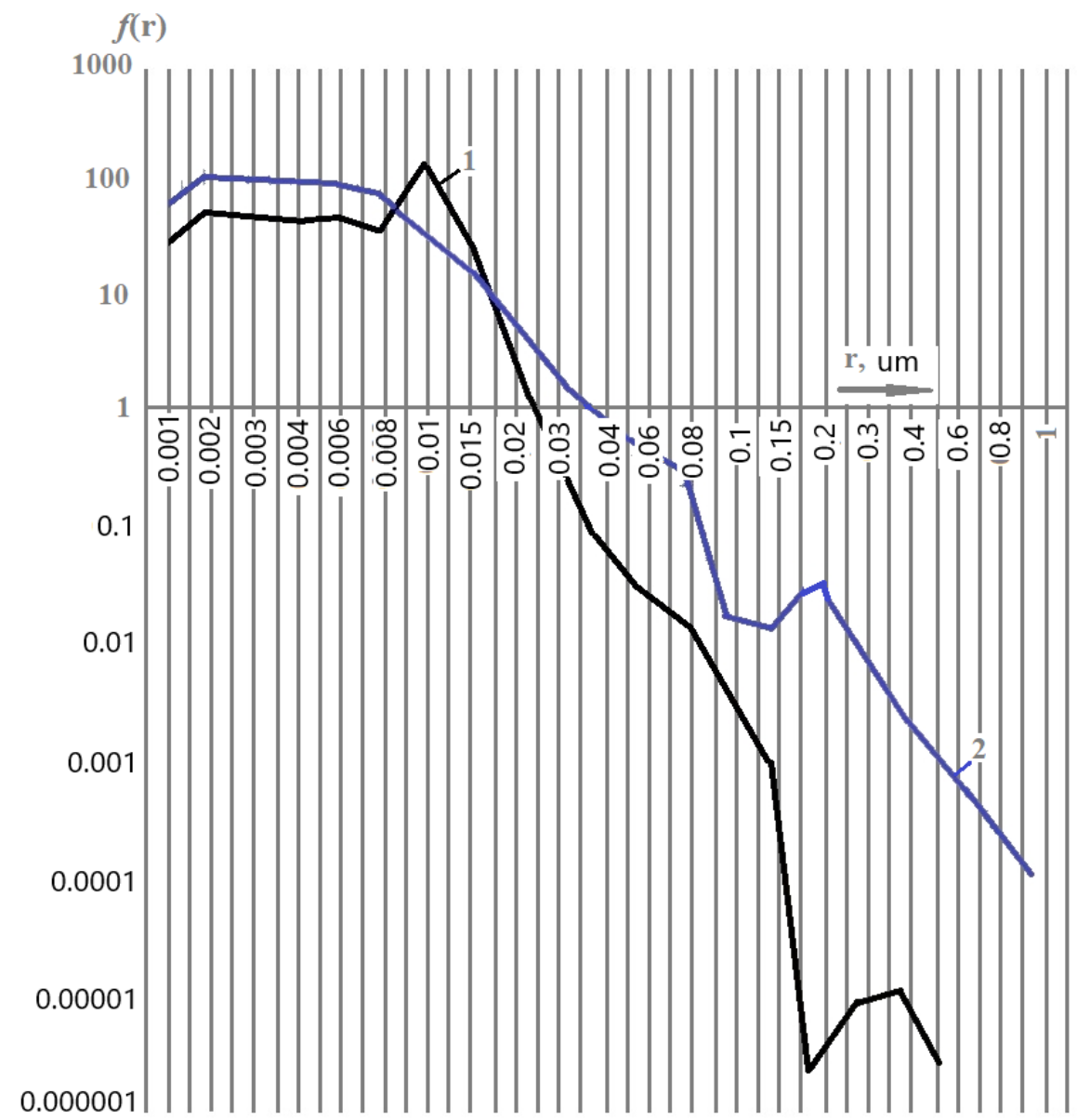

Fig. 4. The distribution function $\mathrm{f}(\mathrm{r})$ of the number of particles in size for the products of combustion: 1 - gasoline engine; 2 diesel engine.

The greenhouse effect of the particulate sol can be significant and it is mainly due to the absorption of solar radiation by the anthropogenically disturbed atmosphere. With the presence of soot particles and clouds that capture the fine particulate sol the albedo of the atmosphere for solar radiation is reduced. On the other hand, the soot sol captured by clouds reduces the lifetime of the clouds. At present international cooperation is widespread in solving problems of fine-structure spectroscopy, see for example [13-15].

The methane trend of climate warming can be significantly influenced by permafrost melting leading to the destruction of methane hydrates [16] and its emissions to the atmosphere. The performed calculations show that the temporal increase in the concentration of methane in the atmosphere from 1964 to the present time leads to an increase in the mean global temperature at the Earth surface by $\Delta \mathrm{T} \approx 0.4 \mathrm{~K}$.

\section{References}

1. N.I. Moskalenko, S.O. Mirumyants, N.F. Loktev, R.Sh. Misbakhov, Equilibrium and non-equilibrium radiation processes: high-temperature environments, radiative heat transfer, 264 (2014)
2. N.I. Moskalenko, S.O. Mirumyants, S.N. Parzhin, I.R. Dodov, Spectral apparatus with a cryogenic, highthroughput, multipass gas cell for studies of radiation absorption by gaseous media, Journal of Applied Spectroscopy, 83(5), 868-871 (2016)

3. N.I. Moskalenko, S.N. Parzhin, M.S. Khamidullina, Spectrometry of gaseous media using a two-beam installation based on the IKS-24 spectrophotometer, Izvestia vuzov Energy problems, 5, 99-109 (2016)

4. N.I. Moskalenko, S.O. Mirumyants, S.N. Parzhin, I.R. Dodov, Measurement system for study of absorption spectra of gaseous media at high pressures, Journal of applied spectroscopy, 83(3), 449-453 (2016)

5. O.V. Zotov, Measurement of high-discharged solar IR absorption spectra of the atmosphere along oblique paths, Transactions of the VII-th all-union symposium on molecular spectroscopy of high and super high resolution, 2, 93-97 (1986)

6. N.I. Moskalenko, R.Sh. Misbakhov, I.Z. Bagautdinov, N.F. Loktev, I.R. Dodov, Determination of ingredient composition of atmospheric emissions of the turbojet engine combustion gases by the fine-structure spectroscopy, Russian aeronautics, 59(3), 419-425 (2016)

7. N.I. Moskalenko, M.S. Khamidullina, Ya.S Safiullina, I.R. Dodov, Study of spectral optical 
characteristics and microstructure of anthropogenic sols, Atmospheric and oceanic optics, 35(8), 628-633 (2018)

8. N.I. Moskalenko, I.R. Dodov, Kayumova, A.R. Khaertdinova, M.S. Khamidullina, E.G. Sheshukov, Modelation of the radiation characteristics of the gas phase combustion products based on high temperature atlases of spectral line parameters, Izvestia vuzov Energy problems, 11-12, 65-80 (2018)

9. N.I. Moskalenko, Identification of ingredients and determination of the ingredient composition of atmospheric emissions and combustion products by the method of fine structure spectroscopy, Alternative energy and ecology, 2, 43-54 (2010)

10. World meteorological organization 2018 https://www.wmo.int/pages/index en.html

11. K.Ya. Kondratiev, N.I. Moskalenko, The greenhouse effect of the atmosphere, 260 (1984)

12. N.I. Moskalenko, Ya.S. Safiullina, M.S. Sadykova, Zonal modeling of the greenhouse effect of the atmosphere and anthropogenic climate change, Alternative energy and ecology, 3, 48-59 (2014)

13. L.S. Rothman, The HITRAN 2012 molecular spectroscopic database, Journal of quantitative spectroscopy and radiative transfer, 130, 4-30 (2013)

14. C. Hill, HITRAN online an online interface and the flexible representation of the spectroscopic HITRAN data base, Journal of quantitative spectroscopy and radiative transfer, 177, 15-42 (2016)

15. L.E. Gordon, The HITRAN molecular spectroscopy database, Journal of quantitative spectroscopy and radiative transfer, 203, 3-69 (2017)

16. A.B. Sherstyukov, Permafrost of Russia in conditions of global climate warming, Evolution and dynamics of geosystems, 4, 8-11 (2007) 